PALAVRAS. Revista de Epistemología, Metodología y Ética del Psicoanálisis

\author{
ISSN: 2468-9831 \\ www.revistas.unlp.edu.ar/palavras \\ palavras@outlook.com.ar \\ Argentina
}

\title{
LOS VALORES EN EL PSICOANÁLISIS: ¿UNA REFLEXIÓN RESISTIDA?
}

\author{
DOI $10.24215 / 24689831 \mathrm{e} 017$
}

Lucía Soria

\begin{abstract}
This paper addresses the issue of valorative assumptions (epistemic and non epistemic values) within psychoanalytic theory and practise, in order to suggest that it should be taken into consideration in current discussions regarding "epochal changes" and psychoanalysis. It begins with a review of some semantic features found in freudian concept of "resistance", that are then put into dialogue with Derrida's questions about nowadays "resistances of psychoanalysis". It is presented the idea that one of the fields in which these resistances take place is that of science. It is argued that in mainstream psychoanalysis in our context, the most frequent understanding concerning scientific activity links it to the positivist model, without references to other ways of understanding scientific activity and its ontological, methodological and epistemological implications. It is proposed that, as a result, two ways of understanding the relationship between psychoanalysis and science are strongly held. A necessary and mutual exclusion between science and psychoanalysis (in order to conserve the latter's specificity), or the urge to respond to this scientific model as a way of legitimizing its epistemological status. Finally, some theoretical and methodological tools developed by critical approaches in psychology and psychoanalysis are considered as a possible contribution to this debate and as a way of reintegrating value assumptions (in its subjective and collective dimensions) into psychoanalytic theory and practise.
\end{abstract}

Key words: Psychoanalysis, Values, Science, Critical

Psychology.

\section{Resumen}

El presente escrito aborda la cuestión de los presupuestos valorativos (epistémicos y no epistémicos) en la teoría y práctica psicoanalíticas, proponiendo localizarlos en el centro de los debates actuales respecto del lugar del psicoanálisis ante los "cambios de época". Para ello, se parte de un recorrido por algunos enveses semánticos presentes en la noción freudiana de "resistencia", que se ponen en diálogo con las preguntas de Derrida acerca de las "resistencias del psicoanálisis" en los tiempos que corren. Al respecto, se presenta la idea de que uno de los ámbitos en que se configuran estas resistencias es aquel vinculado al estatuto del psicoanálisis frente al campo de las ciencias. Se argumenta que las posiciones más frecuentemente esgrimidas en las corrientes psicoanalíticas tienden a perpetuar una concepción de la ciencia fuertemente asociada al modelo positivista, sin considerar las propuestas alternativas a esta concepción tradicional, ni a sus presupuestos ontológicos, epistemológicos y metodológicos. Como resultado, las corrientes hegemónicas suelen, o bien sostener la mutua exclusión entre ciencia y psicoanálisis (en un intento por defender su especificidad), o bien considerar necesaria una aproximación a aquellos parámetros de cientificidad para brindar validez a la disciplina. Finalmente, se propone hacer lugar a algunas herramientas desarrolladas por las perspectivas críticas en psicología y en psicoanálisis, como vía posible para reintroducir las dimensiones valorativas (subjetivas y colectivas) constitutivas de las teorizaciones psicoanaliticas, sin hipotecar por ello su racionalidad ni legitimidad epistémicas.

Palabras claves: Psicoanálisis, Ciencias, Valores, Psicología Crítica.

\section{Cómo citar este artículo:}


PALAVRAS. Revista de Epistemología, Metodología y Ética del Psicoanálisis Número 3 | Diciembre de 2017

Soria, L. (2017). Los valores en el psicoanálisis: ¿una reflexión resistida? Palavras. Revista de Epistemología, Metodología y Ética del Psicoanálisis, 3, 27-45. Recuperado de www.revistas.unlp.edu.ar/palavras 


\section{LOS VALORES EN EL PSICOANÁLISIS: ¿UNA REFLEXIÓN RESISTIDA?}

Lucía Soria ${ }^{*}$

\section{Un rodeo a la cuestión}

La noción freudiana de "resistencia" (Widerstand) hace su aparición muy temprano en los recorridos teórico-clínicos del autor. Recordemos que Freud la hace equivaler inicialmente a la manifestación actual -en el terreno de la cura- de la misma fuerza de repulsión que operó al momento del conflicto psíquico - es decir, de la defensa- y cuya tendencia no es otra que oponerse a la evocación de determinadas representaciones afectivamente displacenteras. De alli que la resistencia sea caracterizada como un fenómeno asible clínicamente, a diferencia de la defensa, hipótesis teórica por excelencia, sólo constatable por sus efectos. Freud va a conservar y de hecho pluralizar a lo largo de su obra este concepto ${ }^{2}$, entendido de manera global y vehemente como "todo lo que perturbe la prosecución del trabajo [analítico]" (Freud, 1900/1992, 511). Se trata, por supuesto -aclara el autor-, de una definición que tiene el valor de un principio técnico y no de una sentencia indiscutible. Sostengamos, al respecto, esta máxima, fundamental a la técnica psicoanalítica: "E1 psicoanálisis es desconfiado, y con razón" (Ibíd., 511).

Algún tiempo después, Freud imprime un vuelco interesante a la cuestión, al comenzar a hablar en sus trabajos de las resistencias al psicoanálisis o contra el psicoanálisis. Un salto conceptual nada evidente, en la medida en que con esta extensión del término se desdibuja el asiento clínico, la “patria de origen" tan cara al método freudiano (Freud, 1933/1992, 140)³.

1* Facultad de Psicología de la Universidad Nacional de La Plata, Argentina. Mail: 1soria@psico.unlp.edu.ar

2 Propuesta que deriva hacia 1926 en la distinción, no exenta de problemas, de cinco resistencias clasificadas de acuerdo a su referencia a alguna de las instancias de la segunda tópica psíquica (Freud, 1926/1975, 147-150).

${ }^{3}$ Señalemos que este uso del concepto resulta complejo -y Freud se percata de ello- ya que abona la frecuente objeción de sus críticos según la fórmula "heads I win, tails you 
No obstante, Freud encuentra sobradas razones para sostenerlo. Desde los tiempos inaugurales del psicoanálisis, habia tomado registro de las negativas que recibía, de las severas criticas y los cuestionamientos que se dirigian a sus ideas, así como a su persona. Con su peculiar agudeza, convierte estas "tormentas de indignada repulsa" en materia de reflexión y les consagra varios pasajes, destinados a elucidar los factores cooperantes en estas resistencias. Es claro que el psicoanálisis tiene sus dificultades, reconoce. Sus piezas teóricas fundamentales son de difícil transmisión, más aún, al estar imposibilitada la participación de terceros oyentes en un análisis. Además -continúa Freud-, frente a su contemporaneidad no eran inesperadas reacciones adversas dado que sus enunciados conmueven muchas verdades establecidas y marcos de inteligibilidad presupuestos, al contrariar las doctrinas médicas y filosóficas vigentes (Freud, 1925a/1992).

Sin embargo, estas razones, que justificaban la renuencia intelectual, no resultaban suficientes para explicar las manifestaciones de escarnio, indignación y virulenta enemistad que se le dirigían. Tampoco alcanzaban para dar cuenta de los verdaderos coup de foudre que con frecuencia encontramos ante las teorias: flechazos a primera vista o conversiones instantáneas, tan fulminantes como aquellos odios encarnizados.

Es a partir de considerar estos matices que Freud conjetura factores de valencia afectiva entre los fundamentos del rechazo al psicoanálisis. Y entiende que las desautorizaciones intelectuales no necesariamente están exentas de motivos afectivos, que se muestran a nivel de los individuos pero también desde el lugar de los prejuicios asumidos y arraigados colectivamente ${ }^{4}$. Así, el rechazo al psicoanálisis puede estar supeditado al horror a verse reflejado en los rasgos que devuelve el espejo de la teoría, a

loose" (si es cara yo gano, si es ceca vos perdés): si el paciente acepta la interpretación, es correcta, si no la acepta, no es más que un signo de la resistencia y, por lo tanto, la interpretación es correcta (Cf. Freud, 1937/1975).

\footnotetext{
4 "Así como hacemos del individuo nuestro enemigo descubriéndole lo reprimido en él, la sociedad no puede responder con solicitud simpática al intransigente desnudamiento de sus perjuicios e insuficiencias; puesto que destruimos ilusiones, se nos reprocha poner en peligro los ideales" (Freud, 1910/1992, 139).
} 
la posibilidad de encontrarse con lo activamente desconocido sobre uno mismo (Freud, 1913/1991, 214). Al respecto, usualmente, se hace referencia a las tres heridas que la ciencia habria propiciado en el amor propio de la humanidad, según la ya célebre formulación del autor, donde el psicoanálisis habría asestado el golpe último, la "afrenta psicológica" que devela que la conciencia no es soberana absoluta en el alma (Cf. Freud, 1925a/1992, 234-35; 1917/1975, 131-135).

No obstante los obstáculos presentados, respecto al horizonte de posibilidad del saber psicoanalítico, Freud hace suya una esperanzada apuesta a la temporalidad:

Las más graves verdades terminarán por ser escuchadas y admitidas después que se desfoguen los intereses que ellas lastiman y los afectos que despiertan. Siempre ha sido así hasta ahora, y las indeseadas verdades que los analistas tenemos para decirle al mundo hallarán el mismo destino. Sólo que no ha de acontecer muy rápido; tenemos que saber esperar $(1910 / 1992,139)$.

Más allá del caso específico, resulta en un todo interesante indagar la lectura que Freud propone de algunos posicionamientos subjetivos y colectivos ante la novedad y ante los desarrollos en las ciencias. Sobre este último punto, sostiene que no hay lugar para el horror a lo nuevo en la empresa científica, que por su permanente incompletud e insuficiencia, la ciencia está condenada a confiar en nuevos descubrimientos y concepciones (1925a/1992, 227). Si estamos de acuerdo en que un escepticismo metódico debiera caracterizar el empeño del investigador sostiene-, se pueden hallar por ocasiones caracteres menos saludables, en que este escepticismo se exacerba, "se pone rígido frente a lo nuevo que llega, en tanto tiene por sacrosanto a lo ya consabido y creído, contentándose con desestimar aquello, aun antes de someterlo a indagación" (1925a/1992, 227). Es interesante que pueda acompañar esa apreciación, algunos años después, con un precepto cauteloso: “De ahí que nosotros, los analistas, tenemos especial fundamento para ser cautos en desautorizar tesis nuevas aduciendo el motivo intelectual” (1933/1992, 31). 
En consecuencia, en la propuesta freudiana, la posición ante las nuevas ideas debe ser considerada, como minimo, a la luz de cierta reflexividad. Esto es, no desconocer que los argumentos intelectuales no desandan a priori prejuicios y preconceptos. Por otra parte y en lo que atañe a nuestro punto de interés, el psicoanálisis no queda exceptuado de estas pasiones.

\section{Otra vuelta de tuerca: el trazado de las fronteras}

El itinerario presentado en lo anterior adquiere vitalidad puesto a dialogar con la lectura que Derrida (2000) proponía hace ya varios años acerca de las "resistencias del psicoanálisis". En los llamados Estados Generales del Psicoanálisis, dedicó su exposición a circunscribir lo que entendía como dos formas de resistencia en curso: "La resistencia al psicoanálisis, en el mundo, [y]... la resistencia al mundo, en el interior de un psicoanálisis que resiste también a sí mismo, que se repliega para resistirse, ...para inhibirse a sí mismo, de manera casi autoinmunitaria” (2000, 17).

En su planteo, el filósofo cargaba las tintas sobre lo que denominaba "la frontera" que así quedaba delineada, delimitando un interior y un exterior, supuestos adentro y afuera de "el psicoanálisis". Sobre esa zona limítrofe ubicaba toda la gravitación de "la carga histórica, ética, jurídica o política" (Ibíd., 16), cuestiones ante las cuales señalaba una marcada incomodidad en el discurso psicoanalítico. Sobre esta misma frontera es que entendía, en consecuencia, que debía resolverse la pregunta por el estado del psicoanálisis, marcado por un notorio malestar de época 5 . Teniendo en

\footnotetext{
${ }^{5}$ El título de la conferencia de Derrida, Etats d'âme de la psychanalyse (Estados de ánimo del psicoanálisis), transmite la idea de un peculiar momento anímico de la disciplina, que conserva en francés similitud fonética con la expresión état de l'âme (estado del alma), además de vinculación etimológica.

Por nuestra parte, con la expresión "malestar de época" hacemos referencia al marco en el que se inserta esta presentación y que continúa atravesando los debates psicoanalíticos contemporáneos, donde los llamados "cambios de época" parecen adquirir, cada vez más, carta de ciudadanía en las explicaciones de la disciplina (Cf. C. Escars, et. al., 2015). Al respecto, digamos brevemente que dichas versiones de "la época" no suelen ser revisitadas a partir del enclave cultural y geopolitico latinoamericano. Contra todo pronóstico, las lecturas del psicoanálisis vernáculo parecieran identificarse plenamente con los desarrollos de los países centrales del sistema de producción del capital teórico psicoanalitico.
} 
mente la distinción bifronte adentro-afuera, se preguntaba, a propósito y alrededor del psicoanálisis:

¿Qué es lo que no marcha bien en los aspectos prevalecientes de su discurso, de su práctica, de su hipotética o virtual comunidad, de sus inscripciones institucionales, de sus relaciones con lo que llamábamos no hace mucho la sociedad civil y el Estado, en el trastorno de su sociología, y de manera diferenciada en cada país, en la mutación que afecta la figura de los pacientes y de los terapeutas, en la transformación de la demanda, de la escena y de lo que llamábamos aún ayer la "situación analítica", cuya precariedad y artificialidad histórica recuerdo haber observado hace décadas? (Derrida, 2000, 8).

Este conjunto, a primera vista tan heterogéneo, donde asoman las resistencias subrayadas por Derrida, aúna ámbitos en que el psicoanálisis se ve compelido a pensar su ánima artificial. Es decir, su dimensión de arte factum, de verdadero aparato producido sociohistóricamente. Eso, el psicoanálisis, no lo analiza. ¿Y por qué pensarlo motorizado por una dinámica resistencial? En gran medida, por la respuesta que -según Derrida- se perfila ante estos interrogantes: una preocupación reparadora, restauradora, del psicoanálisis. Gesto que parece también hoy prevalecer, sea en la pluma de los analistas, en las instituciones psicoanalíticas, en las prácticas profesionales orientadas psicoanalíticamente 0 en la enseñanza del psicoanálisis. Lo que se perfila es una política de la resistencia. Resistencia al avance de las tecnociencias, al sujeto posmoderno (que ya no padecería de su deseo y obtendría del mercado objetos-señuelo que le prometen goce a su medida), a la aceleración de los tiempos (en detrimento de la reflexión), a la sutura del inconciente (producto de la embestida de la industria farmacológica). Estas figuras, entre otras varias, emergen como los frentes de batalla ante los que el psicoanálisis no debe deponer armas. En ellas, no obstante, la responsabilidad por el malestar concierne casi en su totalidad al exterior, a lo que desde esa frontera se asoma, amenazante.

En este punto, vale la pena aclarar que ello está lejos de significar que los analistas se desinteresen del contexto. Contrariamente, desde diversas 
latitudes toman partido ante causas de las más variadas, algunas vinculadas al debate en torno a determinados derechos civiles, otras, a coyunturas políticas o electorales, e incluso, frente a la necesidad de reivindicar la validez del psicoanálisis para el tratamiento de determinadas presentaciones clínicas. Muchos de estos posicionamientos, problematizaciones e intervenciones de los psicoanalistas en los debates contemporáneos respecto de lo colectivo, de la cultura y de las condiciones socioeconómicas de nuestras sociedades, resultan oportunos y de interés. Sin embargo, si lo que unifica a gran parte de estos esfuerzos es el énfasis puesto en que el psicoanálisis no sea anegado por el exterior, lo que no se pone en cuestión es la existencia (o al menos, el trazado) de la frontera.

Subyace a la reflexión psicoanalítica (al menos, en su variante hegemónica) un posicionamiento que tiende a distinguir estáticamente entre lo que compete al terreno propiamente analitico y aquello que, al modo de variante cultural, coyuntural incluso, refiere al contexto y opera como un punto de exterioridad. De este modo, pierden envergadura las discusiones y reflexiones acerca de los presupuestos valorativos tanto epistémicos, como no epistémicos (éticos, políticos, de género, de clase) que se juegan en los postulados teóricos y en los constructos conceptuales psicoanalíticos. En todo caso, se puede considerar que estas variables intervienen en los usos del saber, en lo que se hace "con" el psicoanálisis, pero no así en el corpus teórico ni en los principios técnicos.

Contrariamente, en lo que sigue, se intentará poner en evidencia cómo las posturas típicas frente a la discusión acerca del estatuto científico del psicoanálisis (o bien de las articulaciones entre ciencia y psicoanálisis), desconocen de manera insistente los desarrollos críticos de la concepción tradicional de ciencia. Esto no sólo tiene por correlato una comprensión sumamente empobrecida de la epistemología y metodología científicas en general (al prolongar una visión de la ciencia que ha perdido vigencia aceleradamente desde hace varias décadas), sino además perpetuar la inviabilidad de la discusión, al simplificarla en extremo.

\section{Los ideales heredados y la resistencia a la reflexión}


Sin desconocer los bemoles que se dejan oír en las controversias en torno al estatuto científico del psicoanálisis (que exceden ampliamente las posibilidades y los propósitos del presente escrito), es posible referirse a posiciones típicamente esgrimidas por aquellos que sostienen un necesario distanciamiento entre psicoanálisis y actividad cientifica. Consideremos dos presentaciones de esta cuestión. En primer lugar, un trabajo de Carlo Strenger (2013), autor cuya producción tiene gran circulación en el ámbito del psicoanálisis angloparlante, y que ha tomado como campo de interés estas discusiones desde la perspectiva de la filosofia de las ciencias. En segundo lugar, nos interesa revisar algunas de las conclusiones a las que aborda un grupo de investigadores que se dedica a reconstruir reflexivamente el modo en que se investiga en psicoanálisis en nuestro medio, a partir de explorar prácticas concretas (Azaretto et al., 2014). Ambos trabajos, si bien lejanos en su enclave de producción y en sus horizontes de análisis, resultan coincidentes en el modo en que caracterizan el estado de la discusión respecto al punto de nuestro interés. En el artículo de 2013 titulado "Why psychoanalysis must not discard science and human nature", Strenger sobrevuela el territorio de las discusiones acerca del estatuto del saber psicoanalítico, a partir de historizar algunos de sus derroteros y alertar sobre la persistencia de una distinción esquemática entre dos modelos posibles para pensar la disciplina. Uno, orientado a afianzar la pertenencia del psicoanálisis al terreno y a la metodología de las disciplinas hermenéuticas, interpretativas, sin interés por responder a cánones de cientificidad. El segundo, busca reivindicar su carácter científico, reconciliando al psicoanálisis con el modelo de investigación empírica asociado a los métodos cuantitativos. Ante esta dicotomía, señala el autor, históricamente predominó el primero de los modelos (cuyas propuestas suelen asociarse a las de Jürgen Habermas y Paul Ricoeur), de manera tal que el psicoanálisis mudó gradualmente sus marcos de referencia hacia las humanidades y perdió, paralelamente, sus vínculos con la medicina y las ciencias sociales. Strenger señala que los defensores de esta posición, entre los que se destaca fuertemente, en el ámbito angloparlante, la figura de Irwin Hoffman, entienden al psicoanálisis como "uno de los pocos 
bastiones que restan en nuestra cultura para nociones como las de autonomía, creatividad y subjetividad, y [por ello] debería aferrarse a sus armas" (Strenger, 2013, 198).

A partir de abordar estos modelos, con sus argumentos favorables y contrarios, Strenger concluye que uno de los mayores obstáculos en este debate es la permanencia, en la comunidad analítica, de una representación errada de la ciencia y la actividad científica, concebidas como un proceso mecanizado orientado por una racionalidad técnica. Desde esa perspectiva, al apartarse del proyecto científico, el psicoanálisis no haría más que preservarse de la razón tecnocrática, que persigue poco más que eficiencia en sus resultados. Es justamente aquella distinción la que resulta problemática y tergiversadora, al desconocer otros modos de concebir la cientificidad. Para Strenger, el psicoanálisis contemporáneo se ha defendido del modelo de las ciencias empírico-experimentales (sentido como una amenaza a su especificidad), replegándose en el campo de las hermenéuticas y aferrándose a una reivindicación identitaria y purista. Sin embargo, ello traería aparejado un costo excesivo, al sacrificar su legitimidad científica y aislarlo de otros campos del saber, en lugar de propiciar el diálogo interdisciplinario.

El trabajo colectivo encabezado por Clara Azaretto y Cecilia Ros (2014) brinda interesantes descripciones acerca de las características que adoptan las investigaciones en psicoanálisis en nuestro país, especialmente en el marco de la academia y de las instituciones psicoanalíticas. Los autores explican que a partir de abordar los objetos de estudio que aquellas circunscriben, los criterios que dan fundamento a las decisiones metodológicas y epistemológicas adoptadas, y la estructuración de los proyectos de investigación, resulta posible inferir el modelo de ciencia con el cual se dialoga en cada caso. Es decir, identificar qué concepciones respecto de la ciencia y la investigación científica operan como presupuestos de base. De estos análisis extraen una conclusión especialmente interesante. Encuentran que tanto en las instituciones psicoanalíticas de orientación lacaniana como en aquellas vinculadas a la Asociación Psicoanalitica Internacional (IPA), existe coincidencia en el modelo de ciencia sustentado. El paradigma que predomina es aquel 
asociado a las llamadas "ciencias duras", a los procedimientos de cuantificación de los resultados, a la replicabilidad de la observación, a la posibilidad de predicción y a la utilización de terminología no ambigua. Se sostiene este modelo, ya sea para oponerse y distanciarse en el modo de investigar y concebir al psicoanálisis (como es el caso más general de las instituciones lacanianas), o bien para adoptarlo como modelo a seguir en la búsqueda de validación y legitimación de la disciplina (como ocurre en muchos proyectos asociados a la IPA). Como resultado, en ambas posiciones (llevadas a sus extremos), las relaciones del psicoanálisis con la ciencia resultan en un malestar permanente, marcado por la insuficiencia o por la incompatibilidad:

En lo que resulta un intento de negociación con lo que se supone que reclama la comunidad científica, cada posición adopta maneras -ninguna del todo eficaz- que le permitan la convivencia con dichas reglas, bajo el supuesto de la "falla" o la "excepción". (Azaretto, et al., 2014, 26).

Dos rasgos resaltan en este paradigma de ciencia: la validación del conocimiento científico resulta estrechamente vinculada a la cuantificación -siguiendo la pretensión de universalidad-, y el concepto de empiria o base empírica queda restringido al de observación (2014, 25-29). Paralelamente, no hay lugar en la experiencia científica para el sujeto de la actividad, cuyos rastros anularian la perseguida objetividad. Más aún, no resulta casual que estén prácticamente ausentes referencias a las ciencias sociales o a los enfoques metodológicos por ellas desarrollados. Siguiendo el planteo, lo que resulta así delimitado es un formato, una versión de la ciencia llamada usualmente "cientificismo".

Al abordar algunos matices de esta acepción fuertemente instalada, los autores subrayan que la posición epistemológica más difundida entre las investigaciones que se enmarcan en el psicoanálisis lacaniano está sujeta a la tesis según la cual la ciencia forcluye al sujeto6. Sin embargo -

\footnotetext{
${ }^{6} \mathrm{Si}$ bien puede sostenerse que en Lacan prevalece la interrogación por sobre la respuesta acabada respecto de los vínculos entre psicoanálisis y ciencia (Cf. Lacan, 19641965/2010), de acuerdo al momento de su reflexión en que se detenga el lector, podrá leer esos vínculos en términos de antinomia o de posibilidad a desarrollar. Uno de los axiomas que más se reitera y parece obturar toda discusión, es la sentencia, aquí referida, que afirma que "la ciencia es una ideología de la supresión del sujeto" (Lacan,
} 
agregan-, se sostiene de manera unilateral este "mecanismo", sin distinguir niveles de la discusión (teorías formalizadas, proceso de investigación, cuestiones vinculadas a la técnica o la tecnología), ni considerar enunciados que podrían tomarse como contrarios a esta tesis en otros puntos de la obra del psicoanalista francés $(2014,33)$.

Las frecuentes críticas de los psicoanalistas a la ciencia, según las conclusiones presentadas, están orientadas a la reivindicación de un único modo de producir conocimiento válido y a la legitimidad científica vinculada a los valores de neutralidad, objetividad y fundamento empíricoexperimental. Ahora bien, es al sostener esta versión de la ciencia que resulta posible considerar que el sujeto es suprimido. Justamente, por desconocer la historicidad como dimensión constitutiva del sujeto investigador, de su punto de mira posible, y correlativamente, del objeto de estudio delimitado, también socio-históricamente modelado. Esto no se sostiene si entendemos que el sujeto de la ciencia está condicionado por lo que lo precede, por su comunidad de pertenencia o referencia, por el conjunto de debates y discusiones en las que se inscribe, por los marcos referenciales, las prácticas y las políticas investigativas. Sin embargo, dichas dimensiones resultan imponderables en este modelo de ciencia. Los autores expresan, al respecto:

\begin{abstract}
Abdicar de la historicidad, como condición constitutiva del objeto que se analiza así como del sujeto que opera analizándolo, lleva a discutir con una versión 'cientificista' de la ciencia: como conjunto de prescripciones que describen los atributos de ese tipo de conocimiento -conocimiento racional, sistemático, verificable, exacto y fiable como afirma Bunge (1972)- y no con una versión historicista/constructivista/dialéctica, como práctica social, como discurso (Azaretto, et. al., 2014: 32).
\end{abstract}

Ante las polémicas en torno al estatuto del psicoanálisis, su verificabilidad, legitimidad, eficacia, vemos con frecuencia retornar las dos posturas aquí esbozadas: aquella que sostiene de entrada la incompatibilidad y la mutua exclusión entre ciencia y psicoanálisis, y el no menos problemático bautizó y conceptualizó como "el discurso de la ciencia", restringiendo superficial e inadecuadamente el campo semántico de ambas expresiones. 
esfuerzo por responder a un modelo de cientificidad que opera como lecho de Procusto. En ambos casos, es el mismo paradigma de ciencia aquel que se toma por interlocutor. Entre los factores que concurren en esta dirección, no es menor el peso que, en el psicoanálisis, tiene el principio de autoridad. Se ha configurado una suerte de tradición peculiar, que toma a título de axiomas algunas reflexiones hechas por los analistas "fundacionales" (especialmente, Freud y Lacan), y limita la discusión a la exégesis de los posicionamientos -o sugerencias incluso- que allí se dejan leer.

Si bien las dos posiciones son presentadas como versiones extremadas a los fines expositivos, resulta sumamente infrecuente encontrar otros modos de aproximación al debate que, sin banalizar su complejidad, permitan reintegrar los elementos desatendidos.

\section{Lo desvalorizado en la teoria psicoanalitica}

En el recorrido anterior hemos intentado poner de relieve que la concepción de ciencia prevalente en las discusiones del psicoanálisis hegemónico, no da lugar a la consideración de las marcas subjetivas e históricas en las teorías y prácticas. Sin embargo, el psicoanálisis tampoco ha propiciado otras herramientas que permitan revisar de manera sistemática el estatuto histórico y sociocultural de sus categorías de análisis e intervención. Si bien es posible decir que los esfuerzos emprendidos en la dirección de una reflexión culturalmente sensible han acompañado a la historia de la disciplina ${ }^{7}$, estos proyectos nunca

\footnotetext{
7 Se pueden mencionar aquí desde los empeños de la corriente culturalista en psicoanálisis, de la mano de autores como E. Fromm, K. Horney y H. S. Sullivan, hasta las propuestas de los fundadores del llamado "etnopsicoanálisis", G. Roheim y G. Devereux. En la actualidad, las investigaciones en torno a las narrativas culturales nativas, al rol del contexto en el uso del lenguaje y en las expresiones de los afectos, a la influencia de las experiencias de opresión social y de los estereotipos étnicos o genéricos en los procesos terapéuticos y en las lecturas teóricas, comienzan a aparecer en desarrollos psicoanaliticos en otras latitudes. Se ha propuesto, entre otras cuestiones, una introducción sistemática de cierta "competencia cultural" (cultural competence) en la enseñanza, en las investigaciones y en las prácticas psicoanalíticas, sosteniendo que el psicoanálisis puede al mismo tiempo transformar y ser transformado por el reconocimiento de los aspectos culturales (Cf. Tummala-Narra, 2015).
} 
adquirieron notoriedad en el conjunto de las voces que conforman las orientaciones hegemónicas. Como resultado de estas ausencias, muchos de los presupuestos valorativos que permanecian (o permanecen) desconocidos en el discurso psicoanalítico, se han tornado visibles a partir de las críticas que se formulan desde otros terrenos disciplinares. ${ }^{8}$

Señalemos, a riesgo de simplificar la cuestión, que en este territorio parece configurarse un aspecto resistencial del psicoanálisis, que al menos en su generalidad, se ha encargado de erigir y sostener el objeto mismo de sus críticas. Esta visión estrecha de la actividad científica y de sus productos, resulta desconocedora de otros paradigmas epistémicos, y no resiste a las reconstrucciones que desde la historia de las ciencias, la sociología de las ciencias y las perspectivas de género, entre otros enfoques, se han realizado del devenir de los empeños científicos. De esta manera, nos preguntamos cuán alejados estamos de repetir la clásica maniobra de la resistencia: no mirar a través del microscopio a fin de no ver lo que previamente se había impugnado (Freud, 1925b/1975, 47).

Entendemos que no se trata de intentar encauzar un debate que se habria "descarriado", o de ajustar una terminología imprecisa, sino de preguntarnos qué potenciales aportes pueden hacer al psicoanálisis otras versiones acerca de la participación de las valoraciones epistémicas y no epistémicas en las disciplinas. Y es en este punto que nos parece de gran valor comenzar a transitar algunas de las propuestas enmarcadas en los desarrollos críticos en psicología y psicoanálisis ${ }^{9}$. En primer lugar, porque

\footnotetext{
${ }^{8}$ En este punto, son de mención obligada los esfuerzos de revisión crítica propuestos desde la intersección con las teorías feministas, así como desde las aproximaciones entre la antropología y el psicoanálisis. Estos han orientado la mirada sobre la existencia de valoraciones sesgadas genéricamente y sobre la universalización de presupuestos culturales en el pensamiento psicoanalítico. Para una visión amplia de los desafios desde la mirada del feminismo psicoanalítico puede consultarse D. Tajer (2013); dos fecundos ejemplos de lo segundo son los artículos de F. Benslama (2006) sobre la diferencia sexual en el mundo árabe-musulmán, y de S. Vallon (2010) sobre el cuerpo como una específica ficción occidental asumida y naturalizada por el psicoanálisis.

${ }^{9} \mathrm{Si}$ bien no resulta sencillo brindar una perspectiva sintética de la Psicología Crítica en su diversidad de orígenes, metodologías, objetivos y teorizaciones, siguiendo a Teo (2015) podemos destacar que suele hablarse de un "programa negativo" de la Psicología Crítica, asociado a la crítica específica de la psicología mainstream norteamericana, y de un
} 
estas perspectivas se han dedicado a deconstruir la versión positivista de la ciencia, pero además, porque al hacerlo han reintroducido al sujeto en la discusión.

Desde la versión tradicional, positivista, de la ciencia, en la justificación de una teoría sólo intervienen criterios estrictamente epistémicos y no apreciaciones morales, politicas, de género, etc. Estas últimas pueden ser consideradas, en todo caso, como variables participantes en la gesta de las hipótesis o en la aplicación de los conocimientos a la realidad. Los valores y las preferencias de los investigadores son potencialmente irracionales o afectivos, por lo tanto, es deseable que estos dominios no intervengan en la actividad científica. Contrariamente, las perspectivas críticas proponen, de base, que las teorias y las prácticas están atravesadas subjetiva y colectivamente. En lugar de un discutible intento por desalojar estos presupuestos, la apuesta es tornarlos explícitos, analizarlos críticamente, y poder decidir si sostenerlos o bien concebir posibles alternativas. En esa potencialidad reflexiva, crítica, radica un criterio para ponderar la fortaleza de la teoría ${ }^{10}$.

De esta manera, las marcas de las tradiciones disciplinares no son vistas como una falencia o una debilidad, sino como un valor a tematizar, junto con las posibilidades o no de inserción en las comunidades académicas, las politicas institucionales, las adhesiones a marcos y/o a prácticas compartidas y valoradas, las contradicciones no resueltas por los marcos teóricos, las distintas interpretaciones de un mismo fenómeno propuestas por corrientes diversas, etc.

Estos empeños críticos confluyen en una comprensión de los conceptos psicológicos como social e históricamente producidos y cargados de valores, lo que hace necesario pensarlos vinculados a los efectos que pueden propiciar en los sujetos (o comunidades) a los que se aplican. A su

\footnotetext{
"programa positivo", entendido como las contribuciones a los saberes y a las prácticas contra-hegemónicos y con finalidad emancipadora.

10 A partir de estas propuestas, se entiende a la reflexividad como la "exploración consciente de la manera en que nuestros valores y suposiciones afectan nuestras metas teóricas, nuestra metodología y actividades, y las interpretaciones que realizamos" (Talak, 2014, 12).
} 
vez, dijimos, no se trata de variables que deban ser anuladas metódicamente para que no intervengan en la correcta comprensión de la realidad psicológica o psicosocial, sino que lo central será tematizarlas para tornarlas objeto mismo de análisis. Y ello, nuevamente, obliga a volver la mirada sobre la propia praxis, para reflexionar acerca de los efectos que las categorias, vocablos, asignaciones e intervenciones concretas detentan.

\section{A los fines de concluir}

Uno de los puntos de mayor interés de las perspectivas críticas en psicología y en psicoanálisis consiste en no asumir como algo dado de suyo los propios posicionamientos éticos, políticos y teóricos. El esfuerzo se orientará, entonces, a tornar visibles las consecuencias que esos presupuestos y valoraciones puedan tener en los sujetos intervenidos desde el saber experto, para propiciar, quizá, otros modos de construcción del mismo. Estas dinámicas, a su vez, no pueden ser pensadas por fuera del proceso de psicologización característico de nuestra occidentalidad contemporánea. Las categorías de las ciencias sociales y humanas tienen un estatuto ontológico peculiar, que hace que interactúen con los individuos y las comunidades que pretenden nominar (Rose, 1998; Hacking, 1994). De allí que la noción de praxis, fundamental al psicoanálisis, sirva para destacar la dimensión de toda práctica psi como ético-politicamente orientada desde el posicionamiento teórico mismo, aunque no lo sea solamente desde allí.

Al concebir la praxis vinculándola a los atravesamientos singulares, a las tramas sociales y culturales, pero también a los contextos académicos y profesionales, resulta posible concebir otros modos del quehacer psicoanalítico. Desde este enfoque, lo primero que pondremos en cuestión es la distinción taxativa entre un adentro y un afuera de la disciplina, quirúrgicamente delimitables. No resulta posible sostener la mera exterioridad de aquellos factores que harian al contexto, y que resultarian escindibles de lo psicoanalítico propiamente dicho. Si planteamos que los presupuestos y valores asumidos tienen consecuencias en la formulación y 
en la utilización de categorias de análisis, conceptos y herramientas de intervención, podemos preguntarnos cómo tornarlos representables.

No tematizar las dimensiones ético-politicas, puede conducir a una naturalización del dominio disciplinar, de sus herramientas teóricas y de sus intervenciones prácticas, como también a esencializar y descontextualizar, en nuestro caso, al sujeto destinatario de la práctica psicoanalitica. No debiera ser ajeno a nuestro conocimiento que la teoría y las prácticas psi orientadas psicoanalíticamente sirven de fundamento a gran parte de las discusiones, decisiones (en términos de politicas públicas, de diagnósticos, etc.) y acciones (intervenciones, programas, seguimientos, etc.) en salud mental en nuestro país. En este sentido, por ejemplo, el uso de una categoría diagnóstica para nominar una modalización del padecer subjetivo, afecta el modo en que esa situación es comprendida (como problemática o no, individual, grupal, colectiva, irreversible, atenuable, insalubre, etc.) y, por lo tanto, las propuestas o soluciones, que se puedan ofrecer.

Consideramos que ciertos efectos descontextualizantes en la transmisión y en la comprensión del psicoanálisis, pueden vincularse al modo en que la disciplina ha pensado tradicionalmente su proceder. Es decir, en el esfuerzo por diferenciarse de una versión estereotipada de la ciencia, el psicoanálisis hegemónico puede encontrarse sosteniendo un soliloquio que lo ha apartado de otros modos de concebir la actividad científica, más afines a algunos de sus presupuestos teóricos. Es así que, retomando algunas de las propuestas formuladas a partir de las perspectivas críticas, nos parece que puede relanzarse la pregunta por las circunstancias contemporáneas de la praxis psicoanalitica, a cambio de no desconocer las propias incrustaciones histórico-sociales de la teoría.

\section{Referencias bibliográficas}


Azaretto, C.; Ros C.; Barreiro Aguirre, C.; Wood, L.; Murillo, M.; Estévez, A. \& Messina, D. (2014). Investigar en psicoanálisis. Buenos Aires: JCE Ediciones.

Benslama, F. (2006). El campo sexual en el Islam y la modernidad. En P. L. Assoun \& M. Zafiropoulos (Dir.) Lógicas del sintoma. Lógica Pluridisciplinaria (pp. 227-236). Buenos Aires: Nueva Visión.

Derrida, J. (2000). Los estados de ánimo del psicoanálisis. Lo imposible más allá de la soberana crueldad. Traducido por Escuela de Filosofia Universidad ARCIS. Recuperado de: http://www.ddooss.org/articulos/textos/derrida_psicoanalisis.pdf

Escars, C. (comp.) (2015). Declinaciones del padre. Lecturas psicoanaliticas de la época. Buenos Aires: Letra Viva.

Freud, S. (1900/1992). La interpretación de los sueños. Obras completas de Sigmund Freud, Vol. IV. Buenos Aires: Amorrortu.

Freud, S. (1910/1992). Las perspectivas futuras de la terapia psicoanalitica. Obras completas de Sigmund Freud, Vol. XI (pp. 129142). Buenos Aires: Amorrortu.

Freud, S. (1913 [1911]/1991). Sobre psicoanálisis. Obras completas de Sigmund Freud, Vol. XII (pp. 207-216). Buenos Aires: Amorrortu.

Freud, S. (1917 [1916]/1992). Una dificultad del psicoanálisis. Obras completas de Sigmund Freud, Vol. XVII (pp. 125-136). Buenos Aires: Amorrortu.

Freud, S. (1925a [1924]/1992). Las resistencias contra el psicoanálisis. Obras completas de Sigmund Freud, Vol. XIX (pp. 223-238). Buenos Aires: Amorrortu.

Freud, S. (1925b [1924]/1975). Presentación autobiográfica. Obras completas de Sigmund Freud, Vol. XX (pp. 1-70). Buenos Aires: Amorrortu.

Freud, S. (1926/1975). Inhibición, sintoma y angustia. Obras completas de Sigmund Freud, Vol. XX (pp. 71-154). Buenos Aires: Amorrortu.

Freud, S. (1933 [1932]/1992). 30 a conferencia. Sueño y ocultismo. Obras completas de Sigmund Freud, Vol. XXII (pp. 29-52). Buenos Aires: Amorrortu. 
Freud, S. (1933 [1932]/1992). 34 $4^{\mathrm{a}}$ conferencia. Esclarecimientos, aplicaciones, orientaciones. Obras completas de Sigmund Freud, Vol. XXII (pp. 126-145). Buenos Aires: Amorrortu.

Freud, S. (1937/1975). Construcciones en análisis. Obras completas de Sigmund Freud, Vol. XXIII (pp. 255-270). Buenos Aires: Amorrortu.

Hacking, I. (1994). The looping effects of human kinds. In D. Sperber, D. Premack, \& A. J. Premack (Eds.), Causal cognition: A multidisciplinary approach (pp. 351-382). Oxford, UK: Clarendon Press.

Lacan J. (1973). Psicoanálisis: Radiofonía \& Televisión. Barcelona, Anagrama, 1977.

Lacan, J. (1964-1965). El Seminario 11: Los cuatro conceptos fundamentales del psicoanálisis. Buenos Aires: Paidós, 2003.

Rose, N. (1998). Inventing our selves. Psychology, Power, and Personhood. Cambridge (U.K.): Cambridge University Press.

Strenger, C. (2013). Why psychoanalysis must not discard science and human nature. Psychoanalytic Dialogues: The International Journal of Relational Perspectives, 23 (2), 197-210.

Tajer, D. (2013). Diversidad y clínica psicoanalítica: apuntes para un debate. En A. M Fernández \& W. Siqueira Peres (Eds.). La diferencia desquiciada: géneros y diversidades sexuales, (pp. 123-142). Buenos Aires: Biblos.

Talak, A. M. (2014). Los valores en las explicaciones en psicología. En A. M. Talak (coord.). Las explicaciones en psicología (pp. 147-165). Buenos Aires: Prometeo.

Teo, T. (2012). Philosophical Concerns in Critical Psychology. En D. Fox, I. Prilleltensky \& S Austin (Eds.), Critical Psychology. An Introduction (2nd ed.) (pp. 36-53). London: Sage. Traducción al castellano de Ana María Talak (2015). Cátedra de Psicología II, Facultad de Psicología, Universidad Nacional de La Plata.

Teo, T. (2015). Critical Psychology. A Geography of Intellectual Engagement and Resistence. American Psychologist, 70(3), 243-254.

Tummala-Narra, P. (2015). Cultural competence as a core in emphasis of psychoanalytic psychotherapy. Psychoanalytic Psychology, 32 (2), 275-292. 
Vallon, S. (2010). Cuerpo-sexo-psicoanálisis ¿Libido unlimited? Lapsus calami: El cuerpo y lo sexual. Revista de Psicoanálisis, 1, 29-35. 